\title{
ON THE NIELSEN FIXED POINT THEORY FOR MULTIVALUED MAPPINGS
}

\author{
ZDZISłAW DZEDZEJ \\ Institute of Mathematics, University of Gdańsk \\ Wita Stwosza 57, 80-952 Gdańsk, Poland \\ E-mail: zdzedzej@ksinet.univ.gda.pl
}

\begin{abstract}
We present J. Jezierski's approach to the Nielsen fixed point theory for a broad class of multivalued mappings [Je1]. We also describe some generalizations and different techniques existing in the literature.

1. Notations and definitions. Let $X, Y$ be metric spaces. By a multivalued mapping $\Phi: X \rightarrow Y$ we mean a transformation $\Phi: X \rightarrow 2^{Y}$ with nonempty compact values. Many notions known for singlevalued transformations can be generalized to multivalued mappings. For $A \subset X$ the image of $A$ is the set $\Phi(A)=\bigcup_{x \in A} \Phi(x)$. The set

$$
\Gamma_{\Phi}=\{(x, y): y \in \Phi(x)\}
$$

is called the graph of $\Phi$.

There are several notions of continuity.

Definition 1. The mapping $\Phi$ is lower semicontinuous (lsc) (respectively upper semicontinuous (usc)) if for every open subset $V \subset Y$ the set $\Phi^{-1}(V)=\{x \in X$ : $\Phi(x) \cap V \neq \emptyset\}$ (respectively $\Phi_{+}^{-1}(V)=\{x \in X: \Phi(x) \subset V\}$ ) is an open subset of $X$. If $\Phi$ is both lsc and usc, then we say that $\Phi$ is continuous.

In the singlevalued case these three notions coincide. For basic properties and examples of usc (lsc) mappings we refer the reader to [AC] or [Gor].

In order to have a nontrivial fixed point theory we have to consider special classes of multivalued mappings.

Definition 2. A subset $A \subset X$ satisfies the $\star$-property if it is nonempty, connected and there exists an open neighbourhood $U$ of $A$ such that each loop in $U$ is homotopic (with fixed ends) in $X$ to a constant loop.
\end{abstract}

1991 Mathematics Subject Classification: 55M20.

Research supported by UG grant BW 5100-5-0057-6.

The paper is in final form and no version of it will be published elsewhere. 
Definition 3. A multivalued mapping $\Phi: Y \rightarrow X$ is called an m-mapping iff it is upper semicontinuous and the image of each point has the $\star$-property in $X$.

Let us denote by $\Delta^{n}$ the standard $n$-dimensional simplex and by $\partial \Delta^{n}$ its boundary.

Definition 4. A compact subset $A \subset X$ is $\infty$-proximally connected provided for every $\epsilon>0$ there is $\delta>0$ such that for any map $g: \partial \Delta^{n} \rightarrow O_{\delta}(A)$ there is a map $\tilde{g}: \Delta^{n} \rightarrow O_{\epsilon}(A)$ such that $g(x)=\tilde{g}(x)$ for $x \in \partial \Delta^{n}$ (for any natural $n$ ).

If $X$ is an ANR, then the notion of $\infty$-proximally connected sets is equivalent to the notion of $R_{\delta}$-sets (see e.g. [Gor]) . In particular, AR's are $\infty$-proximally connected.

Definition 5. An usc mapping $\Phi: X \rightarrow Y$ is called a $J$-mapping provided all values $\Phi(x)$ are $\infty$-proximally connected.

2. Reidemeister and Nielsen relations. In this section we assume that a space $X$ is connected and admits a universal covering. Let us fix a universal covering $p: X^{\prime} \rightarrow X$.

Definition 6. A mapping $F^{\prime}: Y \rightarrow X^{\prime}$ such that the following diagram commutes



is called a lift of the mapping $F: Y \rightarrow X$.

The following natural property was proved in [Je1], 1.6.

Theorem 1. Let $F: X \rightarrow X$ be an m-mapping and let $x_{1}, x_{2} \in X^{\prime}$ be two points such that $p\left(x_{2}\right) \in F\left(p\left(x_{1}\right)\right)$. Then there exists a unique m-mapping $F^{\prime}: X^{\prime} \rightarrow X^{\prime}$ for which $x_{2} \in F^{\prime}\left(x_{1}\right)$ and the following diagram commutes:

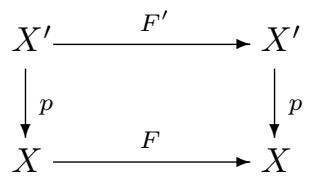

Let us denote by $L(F)$ the set of all m-mappings $F^{\prime}: X^{\prime} \rightarrow X^{\prime}$ making the above diagram commutative. We call the elements of $L(F)$ the lifts of the m-mapping $F$. Recall that all lifts of the identity mapping (called deck transformations) form a group isomorphic to the fundamental group of $X$. Denote this group by $\theta$. If we fix one element $F^{\prime} \in L(F)$, then each lift of $F$ is of the form $\alpha F^{\prime}$, where $\alpha \in \theta$.

Now we can define an equivalence relation $R$ on the set $L(F)$ :

$$
F^{\prime} R F^{\prime \prime} \text { iff } F^{\prime}=\gamma F^{\prime \prime} \gamma^{-1} \text { for some } \gamma \in \theta
$$

This is the so called Reidemeister relation. Let us denote the set of equivalence classes of the Reidemeister relation by $\nabla(F)$. The cardinality of $\nabla(F)$ is independent of the choice of a universal covering of $X$.

DeFinition 7. The number of elements in $\nabla F$ is called the Reidemeister number of the mapping $F$. 
This number is a homotopy invariant ([Je1], 1.12):

THEOREM 2. If two m-mappings are homotopic by an m-homotopy, then their Reidemeister classes are in one to one correspondence.

Now we can define the Nielsen relation in two equivalent ways (comp. [Jia]). Consider the fixed point set of the m-mapping $F: X \rightarrow X$

$$
\text { Fix } F=\{x \in X: x \in F(x)\} \text {. }
$$

Definition 8. Let $x, x^{\prime} \in$ Fix $F$. We say that $x$ and $x^{\prime}$ are Nielsen equivalent iff there exists a lift $F^{\prime} \in L(F)$ such that $x, x^{\prime} \in p\left(\right.$ Fix $\left.F^{\prime}\right)$. We write $x \sim_{N} x^{\prime}$, and denote the quotient set by $\Phi^{\prime}(F)$.

Similarly to the singlevalued case one proves

Theorem 3 (see [Je1]). Let $F: X \rightarrow X$ be an m-mapping. Then

(i) Fix $F=\bigcup_{F^{\prime} \in L(F)} p\left(\operatorname{Fix} F^{\prime}\right)$;

(ii) the sets $p\left(\operatorname{Fix} F^{\prime}\right), p\left(\right.$ Fix $\left.F^{\prime \prime}\right)$ are either equal or disjoint for any $F^{\prime}, F^{\prime \prime} \in L(F)$;

(iii) $p\left(\operatorname{Fix} F^{\prime}\right)=p\left(\right.$ Fix $\left.F^{\prime \prime}\right) \neq \emptyset$ implies that $F^{\prime} \sim_{R} F^{\prime \prime}$.

Consequently there is an injective mapping from $\Phi^{\prime}(F)$ to $\nabla(F)$.

Now we turn to the more popular definition of the Nielsen relation. If $f: X \rightarrow X$ is a singlevalued mapping then two points $x, x^{\prime} \in \operatorname{Fix} f$ are equivalent iff there is a path $\omega: I \rightarrow X$ joining them such that $\omega$ and $f \omega$ are fixed end homotopic. We can reformulate it in the language of the fundamental grupoid. Recall that the fundamental grupoid $\Pi(X)$ is a category. Objects of this category are points of the space $X$ and morphisms from $x$ to $x^{\prime}$ are the fixed end homotopy classes of paths joining these two points. We denote the set of all morphisms between $x$ and $x^{\prime}$ by $\Pi\left(X ; x, x^{\prime}\right)$. Every continuous singlevalued mapping $f: X \rightarrow Y$ induces a functor $\Pi(f): \Pi(X) \rightarrow \Pi(Y)$ by

$$
\Pi(f)(x)=f(x) ; \quad \Pi(f)[\omega]=[f \omega] .
$$

Proposition 4. Let $f: X \rightarrow X$ be a continuous singlevalued mapping. Then two points $x, x^{\prime} \in$ Fix $f$ are equivalent if and only if the mapping

$$
\Pi(f): \Pi\left(X ; x, x^{\prime}\right) \rightarrow \Pi\left(X ; x, x^{\prime}\right)
$$

has a fixed point.

Now let $X$ be a connected, locally pathwise connected, semilocally simply-connected topological space (i.e. admitting a universal covering). Let $A_{0}, A_{1}$ be two subsets of $X$ satisfying the $\star$-property. Then the sets $\Pi\left(X ; a_{0}, a_{1}\right), \Pi\left(X ; a_{0}^{\prime}, a_{1}^{\prime}\right)$ are identified as follows: let $U_{i}$ be a pathwise connected neighbourhood of $A_{i}$ as in the $\star$-property (definition 2) for $i=0,1$. Let $a_{i}, a_{i}^{\prime} \in A_{i}$ and let $\omega_{i}$ be a path in $U_{i}$ joining the points $a_{i}$ and $a_{i}^{\prime}$. We identify $[\alpha] \in \Pi\left(X ;, a_{0}, a_{1}\right)$ with $\left[\omega_{0}^{-1} * \alpha * \omega_{1}\right] \in \Pi\left(X ;, a_{0}^{\prime}, a_{1}^{\prime}\right)$ and define the quotient set

$$
\widehat{\Pi}\left(X ; A_{0}, A_{1}\right)=\bigcup_{\left(a_{0}, a_{1}\right) \in A_{0} \times A_{1}} \Pi\left(X ; a_{0}, a_{1}\right) / \sim
$$


For each $a_{0} \in A_{0}, a_{1} \in A_{1}$ we denote by

$$
i_{a_{0}, a_{1}}: \Pi\left(X ; a_{0}, a_{1}\right) \rightarrow \widehat{\Pi}\left(X ; A_{0}, A_{1}\right)
$$

the natural bijection.

Definition 9. The generalized fundamental grupoid of the space $X$ is the category in which objects are subsets of $X$ satisfying the $\star$-property and $\widehat{\Pi}\left(X ; A_{0}, A_{1}\right)$ is the set of all morphisms between the objects $A_{0}$ and $A_{1}$. We will denote this category by $\widehat{\Pi}(X)$.

Proposition 5. Let $X$ be a connected space admitting a universal covering and let $Y$ be a topological space. Then each m-mapping $F: Y \rightarrow X$ induces a functor

$$
\widehat{\Pi}(F): \Pi(Y) \rightarrow \widehat{\Pi}(X)
$$

which coincides with $\Pi(F)$ when $F$ is a singlevalued mapping.

Proof. We define $\widehat{\Pi}(F)(y)=F(y)$ for each $y \in Y$. Let $[\omega] \in \Pi\left(Y ; y_{0}, y_{1}\right)$. Let us fix a universal covering $p: X^{\prime} \rightarrow X$ and points $x_{0} \in F\left(y_{0}\right), x_{1} \in F\left(y_{1}\right), x_{0}^{\prime} \in p^{-1}\left(x_{0}\right)$. Consider the diagram

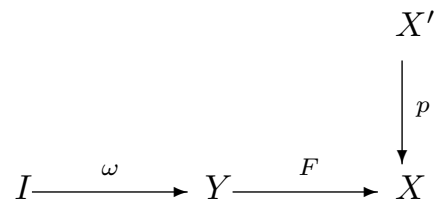

It admits a unique lift $\widetilde{(F \omega)}: I \rightarrow X^{\prime}$ such that $\left.x_{0}^{\prime} \in \widetilde{(F \omega}\right)(0)$. Let $\left\{x_{1}^{\prime}\right\}=\widetilde{(F \omega)}(1) \cap$ $p^{-1}\left(x_{1}\right)$ and let $\tau$ be a path in $X^{\prime}$ joining $x_{0}^{\prime}$ to $x_{1}^{\prime}$. We define

$$
\widehat{\Pi}(F)[\omega]=i_{x_{0}, x_{1}}[p \tau] \in \widehat{\Pi}\left(X ; F\left(y_{0}\right), F\left(y_{1}\right)\right) .
$$

One checks that the definition is independent of the choice of the points $x_{0}, x_{1}, x_{1}^{\prime}$ and the path $\tau$.

Definition 10. Two fixed points $x_{1}, x_{2}$ of the m-mapping $F: X \rightarrow X$ are $i n \sim_{N^{\prime}}$ relation iff the maps

$$
\widehat{\Pi}(F), i_{x_{1}, x_{2}}: \Pi\left(X ; x_{1}, x_{2}\right) \rightarrow \widehat{\Pi}\left(X ; F\left(x_{1}\right), F\left(x_{2}\right)\right)
$$

have a coincidence point.

THEOREM 6 ([Je1], 3.12). The above relation $\sim_{N^{\prime}}$ is equal to $\sim_{N}$.

Equivalence classes of the above relation are called Nielsen classes of the mapping $F$.

3. The Nielsen number. In order to define the Nielsen number one has to define the notion of an essential class. The best way to do it is to use a fixed point index. Therefore we have to consider those classes of m-mappings which admit an index theory.

Let $X$ be a metric ANR.

Definition 11. An m-mapping $F: X \rightarrow X$ is called a Nielsen m-mapping if the image of each point is a Q-acyclic continuum and the image $F(X)$ is relatively compact in $X$. 
For the above class of mappings there exists a fixed point index theory satisfying all the standard properties (see e.g. [Dz1]).

Let $F: X \rightarrow X$ be a Nielsen m-mapping and let $A$ be one of its Nielsen classes. Choose an open set $U \subset X$ such that $U \cap$ Fix $F=A$.

Definition 12. The class $A$ is essential provided $i(X, F, U) \neq 0$. The number of all essential classes is called the Nielsen number of $F$ and is denoted by $N(F)$.

The proofs of two basic properties of the Nielsen number are standard.

Theorem 7 ([Dz1], 9.15). If $H: X \times I \rightarrow X$ is a homotopy in the class of Nielsen m-mappings, then $N\left(H_{0}\right)=N\left(H_{1}\right)$.

Theorem 8 ([Dz1], 9.13). A Nielsen m-mapping $F: X \rightarrow X$ has at least $N(F)$ fixed points.

4. Approximation approach. Observe that the property from Definition 4 is stronger than the $\star$ property if $X$ is an ANR. Therefore our definition of the Nielsen relation works for the class of $J$-mappings. Roughly speaking, mappings of this class can be approximated on the graph by singlevalued transformations, so the Nielsen number of a $J$-mapping is just the Nielsen number of its sufficiently close approximation. This approach has been used in $[\mathrm{KrM}]$ for $J$-mappings and also for their finite compositions.

Let $X, Y$ be two metric spaces. For $\Gamma \subset X \times Y$ we will denote by $O_{\epsilon}(\Gamma)$ the $\epsilon$ neighbourhood of $\Gamma$ in $X \times Y$ with the "maximum" metric.

Definition 13. A continuous mapping $f: X \rightarrow Y$ is an $\epsilon$-approximation of a multivalued mapping $F: X \rightarrow Y$ provided

$$
\Gamma_{f} \subset O_{\epsilon}\left(\Gamma_{F}\right)
$$

Theorem 9. Let $X$ be a compact $A N R$ and $F: X \rightarrow Y$ a J-mapping. Then for any $\epsilon>0$ there exists an $\epsilon$-approximation of $F$. Moreover, for each $\delta>0$ there exists an $\epsilon$ such that any two $\epsilon$-approximations of $F$ are homotopic by a homotopy $H$ such that $H_{t}=H(\cdot, t)$ is a $\delta$-approximation of $F$ for every $t \in I$.

For the proof we refer the reader to [Gor], [GGK]. We now apply Theorem 9 to define a fixed point index (a Nielsen number) of $\mathrm{F}$ to be the index (the Nielsen number) of a sufficiently fine approximation of $\mathrm{F}$. This definition does not depend on the choice of the approximation $([\mathrm{KrM}], 6.5)$.

REMARK 1. There are examples in $[\mathrm{KrM}]$ showing that if one defines the Nielsen number in an analogous way for compositions of $J$-mappings dropping out the assumption of the type $\star$ then the numbers obtained may depend on the way of composition.

5. Other classes of mappings. There were some attempts to consider other classes of mappings for Nielsen theory (see [Dz1-2], [Mas], [S1-4]). If we do not assume that images of points are connected, then we have to consider only continuous (i.e. both usc and lsc) mappings, as the following simple example shows. 
EXAmple 1. The map $F:[-1,1] \rightarrow[-1,1]$ given by

$$
F(x)=\left\{\begin{array}{ccc}
\{x+1,1\} & \text { if } & x<0 \\
\{-1,1\} & \text { if } & x=0 \\
\{-1, x-1\} & \text { if } & x>0
\end{array}\right.
$$

is usc and has no fixed points.

One can use the following observation of S. Banach.

Proposition 10. Let $F: X \rightarrow Y$ be a continuous mapping such that for each point $x \in X$ the image $F(x)$ consists of exactly $n$ components. If $X$ is path connected and simply connected then the graph $\Gamma_{F}$ has exactly $n$ components and consequently $F$ splits into $n$ disjoint usc mappings.

This is true, in particular, for $n$-valued continuous mappings. If we assume that the components of images of points are $Q$-acyclic continua satisfying the $\star$-property, then we are able to prove a version of Thm. 1 (see [Dz1], 9.4). Therefore one can repeat the definition of Nielsen relation and Nielsen number after Section 2 and 3 (see [Dz1] for details).

In general it is hard to expect minimum theorems for multivalued mappings. But for $n$-valued continuous mappings the following theorem is true.

THEOREM 11 (H. Schirmer [S2]). If $X$ is a compact, connected manifold of dimension at least 3 and $F: X \rightarrow X$ is an n-valued continuous mapping, then there exists an $n$ valued mapping $G$ homotopic to $F$ such that the number of fixed points of $G$ is equal to $N(F)$.

One could try to extend the theory to finite-valued continuous mappings. Unfortunately even the Lefschetz fixed point theorem is not true then. In fact in [Je2] an example was given of a continuous mapping with values which are either 1- or 2- or 3-point sets from the two-dimensional disc onto itself without fixed points. However, if we restrict our attention to simpler combinations (e.g. if the images of points have either one or $n$ components) then the index theory works (see [Dz1]). Such mappings determine continuous mappings into symmetric products. This approach has been used to build a Nielsen theory in [Mas], [Dz2], [S3-4]. But the following observation was made in [Mik]. Recall that the symmetric product $X_{n}$ is the orbit space of the action of the symmetric group $S(n)$ on the $n$-fold Cartesian product $X^{n}$ by permutation of coordinates.

Proposition 12. If $X$ is a pathwise connected space, then every symmetric product mapping $f: X \rightarrow X_{n}$ has at most one fixed point class.

This is true also in a multivalued setting (see [Dz2]). Using this fact the following minimum theorem has been proved by H. Schirmer [S4].

THEOREM 13. Let $X$ be a compact, connected manifold of dimension at least 3, and $F: X \rightarrow X$ a continuous mapping with images of points being either singletons or two-point sets. Then there exists a mapping $G$ of the same type, which is homotopic to $F$ with only one fixed point if the Lefschetz number of $F$ is non-zero, and fixed point free otherwise. 


\section{References}

[An] J. Andres, Multiple bounded solutions of differential inclusions: The Nielsen theory approach, Preprint (1997).

[AC] J. P. Aubin and A. Cellina, Differential Inclusions, Springer-Verlag, 1984.

[Bro] R. F. Brown, The Lefschetz Fixed Point Theorem, Scott, Foresman \& Co., Glenview Ill., 1971.

[Do] A. Dold, Lectures on Algebraic Topology, Springer-Verlag, 1972.

[Dz1] Z. DzedzeJ, Fixed point index theory for a class of non-acyclic multivalued maps, Dissertationes Math. 253 (1985).

[Dz2] Z. DzEDZEJ, On Nielsen and Reidemeister relations for set-valued symmetric product maps, CRM Barcelona 76 (1989), 1-8.

[Gor] L. GóRniewicz, Topological approach to differential inclusions, in: Topol. Methods in Diff. Equations and Inclusions, A. Granas and M. Frigon (eds.), NATO ASI 472, 129-190.

[GGK] L. Górniewicz, A. Granas and W. Kryszewski, On the homotopy method in the fixed point index theory for multivalued mappings of compact ANR's, J. Math. Anal. Appl. 161 (1991), 457-473.

[Je1] J. JEzIERski, The Nielsen relation for multivalued maps, Serdica 13 (1987), 174-181.

[Je2] J. JezIERski, An example of finitely-valued fixed point free map, Zesz. Nauk. IM UG 6 (1987), 87-93.

[Jia] B. JiAng, Lectures on Nielsen Fixed Point Theory, Contemp. Math. 14, AMS, Providence, R.I., 1983.

[KrM] W. Kryszewski and D. MikLaszewski, The Nielsen number of set-valued maps. An approximation approach, Serdica 15 (1989), 336-344.

[Mas] S. Masin, On the fixed point index and Nielsen fixed point theorem for symmetric product mappings, Fund. Math. 102 (1979), 143-158.

[Mik] D. MikLASZEWSKI, A reduction of the Nielsen fixed point theorem for symmetric product maps to the Lefschetz theorem, Fund. Math. 135 (1990), 175-176.

[S1] H. SCHIRMER, An index and a Nielsen number for n-valued multifunctions, Fund. Math. 124 (1984), 207-219.

[S2] H. Schirmer, A minimum theorem for $n$-valued multifunctions, Fund. Math. 126 (1985), 83-92.

[S3] H. Schirmer, A fixed point index for bimaps, Fund. Math. 134 (1990), 93-104.

[S4] H. Schirmer, The least number of fixed points of bimaps, Fund. Math. 137 (1991), $1-8$. 